\title{
A Survey on Image Retrieval Techniques
}

\author{
Lalitha $\mathrm{K}^{\mathrm{a}}$ and Murugavalli $\mathrm{S}{ }^{\mathrm{b}}$ \\ ${ }^{a}$ Research scholar (Anna University), Assistant Professor, Panimalar Engineering \\ College, Chennai \\ ${ }^{b}$ Professor \& Head, Deptof CSE, Panimalar Engineering College
}

\begin{abstract}
In the recent years, with the rapid development of technologies production, digital images tend to grow in exponential order. This makes the challenge in retrieving image. Image retrieval plays a vital role in various fields such as Medical, Tourism promotion, Forensics Lab etc. In order to handle these rapid growth efficient images retrieval methods need to be developed. This paper is presented with the survey of different Image retrieval techniques which used various techniques from visual features to the latest deep learning with Convolutional Neural Network(CNN) which become the best approach for image retrieval with number of layers applicable for large database. This survey concretes the way to understand the processing methods of Content Based Image Retrieval (CBIR).
\end{abstract}

Keywords.Image Retrieval, $\mathrm{CNN}$

\section{Introduction}

Due to the rapid evolution of technology, usage of internet, smart phones and digital cameras get increased which leads to the exponential growth in the Image generation. Researchers are attracted with the efficient image retrieval methods to retrieve relevant image from the large database. It is essential for every image retrieval techniques to explore and organize the images that are equal to the query image given by the users.Image retrieval methods started with traditional key word search. Due to the least availability of keyword and inconsistency of text and image lead to the failure of traditional system. The Content Based Image Retrieval (CBIR) method is the most significant methods in image retrieval which overcomes the difficulties of traditional method.In CBIR Input is given as image and it matches the input with the database in terms of feature vectors to produce similar images. Low -level features like color, shape,spatial layout and texture and global features are automatically mined from the image.

Initially Color paid a major role in differentiating an Image[12]. Later it was found that shape can also be considered to retrieve similar images. Due to some drawbacks of previous method made the texture feature important to search accurate result. As a refinement for CBIR combination of color, texture and shape are used for image retrieval. Color moments, color Histogram,

\footnotetext{
${ }^{1}$ LalithaK, Research scholar (Anna University), Assistant Professor, Panimalar Engineering College. E.mail: lali.kalai@gmail.com
} 
Edge Histogram Descriptor and co-occurrence Matrix are used to get contents of images [13].For Large database to retrieve images Content Based Image Retrieval(CBIR) with Graph based Ranking model[1] can be used. The famous Graph based Ranking model, Manifold Ranking(MR) used geometrical structure to rank the data samples. Some of the methods on CBIR like BTC [2], before performing decoding process the reconstructed image is obtained from the feature descriptor from encoded data.

To solve the Machine learning problems and to reduce the storage space several hashing methods have been developed. Similar data with smaller hamming distance were mapped with hash codes. The feature extraction ability is enhanced by Convolutional neural Network. Hashing Performance is boosted by combining with the deep learning methods. DSTH [3] method supports both supervised and unsupervised method to enhance the accuracy of hashing. Importance of CBIR is increasing in the field of education, classification and retrieval of bioinformatics and also in medical field. CBMIR [5] based on hashing with CNN helped medical image retrieval.

\section{Literature Survey}

a) Bin $\mathrm{Xu}$ et al.[1] proposed an Efficient Manifold Ranking (EMR) for Content Based Image Retrieval. EMR addressed the drawbacks of Manifold Ranking (MR) by constructing scalable graph and computing the rank efficiently for large Database.KNearest Neighbor graph and a novel adjacency matrix is considered to speed up the ranking instead of Anchor graph. An approximate method is adopted in EMR to retrieve the out-of-sample efficiently.

b) Jing-Ming Guo and HeriPrasetyo [2] developed a method to generate effective image content descriptor using Ordered-Dither Block Truncation Coding(ODBTC). Bit Pattern Feature and Color Co-Occurrence Feature are extracted from the bitmap images andcolorquantizers which are decomposed from image during the encoding process of ODBTC.

c) Yu Liu et al.[3] suggested a Deep Self-Taught hashing (DSTH) method in order to overcome some of the problems faced by existing image retrieval methods. DSTH is proposed to apply deep hashing on datasets without label. By analysing data itself it generates pseudolabel and used discriminate model to learn the hash function for new data. DSTH used two different deep learning methods to train the hash function in order to deal with the out-of-sample problem. DSTH is also trained for supervised and unsupervised case. The result of this method reduced the time complexity by preserving the accuracy.

d) MudhafarJalilJassimGhrabat et al.[4] proposed a method to solve the current issues such as high time consumption, handling complex queries and low accuracy in content based image retrieval. To improve accuracy and reliability noise is removed using Median filter in pre-processing technique. The features like shape, texture and color are mined from the Pre-processed image including color moment, histogram and coherency vector. Multiple ant Colony Optimization (MACOBTC) is used to select the features and also used to build the classifier using Greedy Learning of Deep BoltzmanMachine(GDBM). The developed method is used in the diagnose section of medical field. 
e) YihengCai et al.[5] proposed a novel Content Based Medical Image retrieval (CBMIR) based on hash coding and Convolutional Neural Network(CNN). CBMIR used Siamese network with pairs of similar or dissimilar images as input and produce the class of images with similar images. A Loss function is reconstructed in training process to make the distinguishable feature vectors and difference between real values output and binary codes is reduced by adding regularization term. The hash code of the database image and binary code of trained network is compared to retrieve image efficiently than other traditional hash codes.

f) Fei Liu et al.[6] proposed a method to overcome the security concern of mobile users in retrieving images. Images from the cloud were retrieved without the user's constant interaction. The deep features of the image are extracted using a pretrained

g) deep Convolutional Neural Network model. Lattice-based homomorphic method concealed the information about neural network. Divide and conquer CNN evaluation protocol and real number computation mechanism is implemented to evaluate deep CNN with more number of inputs in efficient and secured manner. The method to compare two images in the cloud server without knowing their deep features is also developed through image similarity scoring protocol.

h) Ashnilkumar et al.[7] proposed a method which integrates Visual Analytics method for Medical Image retrieval(VAMIR) to overcome the limitation in finding the deviation among how the user perceive the concept of image and search objectiveand how the machine perceive it. VAMIR reduced the semantic gap by allowing the users to explore the feature space to discover the similar images. Instead of rank list from the subset VAMIR allowed to choose similar images by the user from the entire dataset.

i) Haifeng $\mathrm{Hu}$ et al.[8] proposed a method called Metric Learning - Anchor graph hashing(ML AGH) based on semi-supervised Learning which preserved feature space's semantic similarity and data structure's. For a training set, anchor-based similarity graph is constructed by exploiting transformation matrix. By using hinge Loss obtained by the triplet constraint and smoothness of labels, objective function is designed. To facilitate the execution speed of MLAGH penalty factor is designed and transformation matrix is updated using Stochastic Gradient Descent (SGD). The result of this method Minimized the distance among the image and its anchor with similar semantic labels and also preserved structure in the feature space.

j) Shifeng Zhang et al. [9] proposed a deep hashing algorithm which reduces the complexity of previous hashing methods which involved $\mathrm{O}(\mathrm{n} 2)$ data pairs and $\mathrm{O}(\mathrm{n} 3)$ triplets. It reduced the complexity to $\mathrm{O}(\mathrm{n})$ through Unary-Upper bound and aiding the triplet loss and classification based unary loss. The modified Unary Upper Bound, named as Semantic Cluster Unary Loss(SCUL) is used to train the new deep supervised hashing algorithm called Semantic Cluster Deep Hashing(SCDH). By combining Mean Teacher algorithm and SCUL a new Semi Supervised hashing is developed for the large scale database.

k) Shi et al. [10] developed a model to handle the large scale images, extracted image features and learned their binary representation using hashing algorithm. They proposed an objective function and the pairwise matrix with deep learning framework through which binary representations of images are learned. Efficiency of the proposed algorithms is experimented on thousands of histopathology images. 
1) Zhang et al. [11] proposed a new weak semantic consistency constrained (WSCC) to handle the difficulty in managing the labelling process for more number of images. For classification and categorization of digital images supervised learning is the widely used method. Using weak semantic correlations and images within the same class are assigned with same class in the absence of labelledinformation.On the bases of Visual semantics partially labeled images are allotted to various midlevel classes.

\section{Summary}

As per the survey made on various Efficient Manifold Ranking (EMR) [1] which reduced the computational time when compared with LSH and SH, ODBTC [2] indexing produced the better average precision rate of 0.779 when compared with other existing methods. DSTH [3] produced better results for unlabeled data when compared with performance using classification Label. The Experimental result of GDBM [4] on 50,000 datasets provided $25 \%$ improvement in accuracy which made it to be applicable for medical field. Another approach CBMIR [5] framed for medical image retrieval reduced the retrieval time to $382.952 \mathrm{~ms}$ which is comparatively less compared with existing methods like deep hashing. In MLAGH [8] time complexity is linear with number of training samples as the anchor points is less than the training samples. VAMIR [7] made the feature space explored to users who are not well versed in retrieval methods. For mobile users, Content based retrieval methods Run time cost and communication cost are reduced. [6]

\section{Conclusion}

ThisSurvey covered some of the technical aspects of content-based image retrieval techniques. The discussion begins from the previous methods which are based onthe low level features like color, texture, and shape to themost prominent method of latest deep learning with Convolutional Neural network.

\section{References}

[1] Bin Xu, Jiajun Bu, Chun Chen, Can Wang, Deng Cai, and Xiaofei He, EMR - A Scalable Graph-based Ranking Model for Content-based Image Retrieval.in IEEE Transactions on Knowledge and Data Engineering, Vol 27, No. 1, Jan 2015.

[2] Jing-Ming Guo and Geri Prasetyo,Content- Based Image Retrieval Using Features Extracted From Halftoning-Based Block Truncation Coding.in IEEE Transactions on Image Processing, Vol. 24, No. 3 , March 2015.

[3] Yu Liu, Jingkuan song, Ke Zhou, Lingyu Yan, Li Liu, FuhaoZou, and Ling Shao, Deep Self-Taught Hashing for Image Retrieval.in IEEE Transactions on Cubernetics, Vol. 49, No. 6, June 2019.

[4] MudhafarJalilJassimGhrabat ,GuangzhiMa, ZaidAmeenAbduljabbar,Mustafa A. AlSibahee, And SafaJalilJassim, .Greedy Learning of Deep Boltzmann Machine (GDBM)'s Variance and Search Algorithm for Efficient Image Retrieval.in IEEE Access ,Vol 7, 2017.

[5] YihengCai ,Yuanyuan Li, ChangyanQiu, Jie Ma, And XurongGao, Medical Image Retrieval Based on Convolutional Neural Network and Supervised Hashing, IEEE Access, vol 72019.

[6] Fei Liu, Yong Wang, Fan-Chuan Wang, Yong - Zheng Zhang And Jie Lin, Intelligent and Secure Content-BasedImage Retrieval for Mobile Users.in IEEE Access,Vol. 7, 2019.

[7] Ashnil Kumar, Member, IEEE, Falk Nette, Karsten Klein, Michael Fulham, and Jinman Kim, A Visual Analytics Approach Using the Explorationof Multidimensional Feature Spacesfor Content-Based 
Medical Image Retrieval.IEEE Journal Of Biomedical And Health Informatics, Vol. 19, No. 5, September 2015.

[8] Haifeng Hu, Kun Wang, ChenggangLv, Jiansheng Wu, and Zhen Yang ,Semi-Supervised Metric Learning-Based AnchorGraph Hashing for Large-Scale Image Retrieval.in IEEE Transactions On Image Processing, VOL. 28, NO. 2, FEBRUARY 2019

[9] Shifeng Zhang, Jianmin Li and Bo Zhang,Semantic Cluster Unary Loss for Efficient Deep Hashing.in IEEE Transactions on Image Processing, Vol.28, No.6, June 2019.

[10] X. Shi, M. Sapkota, F. Xing, F. Liu, L. Cui, and L. Yang, Pairwise based deep ranking hashing for histopathology image classification and retrieval. Pattern Recognition, vol. 81, pp. 14-22, 2018.

[11] C. Zhang, J. Cheng, and Q. Tian, Unsupervised and semisupervised image classification with weak semantic consistency, IEEE Transactions on Multimedia, 2019.

[12] B.Thenkalvi and S.Murugavalli, Review on CBIR Trends and Techniques to Upgrade Image Retrieval.in International Review on Computers and softwares, Vol 9,July 2014.

[13] R.S.Dubey, R. Choubey, J. Bhattacharga, Multi Feature Content Based Image Retrieval. (IJCSE) International Journal on Computer Science and Engineering, vol. 02, No. 06, 2010 , pp. 2145-2149. 